\title{
Orthogonal Stability of an Additive-Quadratic Functional Equation
}

\author{
Choonkil Park
}

Correspondence: baak@hanyang.ac. $\mathrm{kr}$

Department of Mathematics, Research Institute for Natural Sciences, Hanyang University, Seoul 133-791, Republic of Korea

\section{Abstract}

Using the fixed point method and using the direct method, we prove the Hyers-Ulam stability of an orthogonally additive-quadratic functional equation in orthogonality spaces.

(2010) Mathematics Subject Classification: Primary 39B55; 47H10; 39B52; 46H25.

Keywords: Hyers-Ulam stability, fixed point, orthogonally additive-quadratic functional equation, orthogonality space

\section{Introduction and Preliminaries}

Assume that $\mathrm{X}$ is a real inner product space and $f: X \rightarrow \mathbb{R}$ is a solution of the orthogonally Cauchy functional equation $f(x+y)=f(x)+f(y),\langle x, y\rangle=0$. By the Pythagorean theorem, $f(x)=\|x\|^{2}$ is a solution of the conditional equation. Of course, this function does not satisfy the additivity equation everywhere. Thus, orthogonally Cauchy equation is not equivalent to the classic Cauchy equation on the whole inner product space.

Pinsker [1] characterized orthogonally additive functionals on an inner product space when the orthogonality is the ordinary one in such spaces. Sundaresan [2] generalized this result to arbitrary Banach spaces equipped with the Birkhoff-James orthogonality. The orthogonally Cauchy functional equation

$$
f(x+y)=f(x)+f(y), \quad x \perp y,
$$

in which $\perp$ is an abstract orthogonality relation, was first investigated by Gudder and Strawther [3]. They defined $\perp$ by a system consisting of five axioms and described the general semi-continuous real-valued solution of conditional Cauchy functional equation. In 1985, Rätz [4] introduced a new definition of orthogonality by using more restrictive axioms than of Gudder and Strawther. Moreover, he investigated the structure of orthogonally additive mappings. Rätz and Szabó [5] investigated the problem in a rather more general framework.

Let us recall the orthogonality in the sense of Rätz; cf. [4].

Suppose $X$ is a real vector space (algebraic module) with $\operatorname{dim} X \geq 2$ and $\perp$ is a binary relation on $X$ with the following properties:

$\left(O_{1}\right)$ totality of $\perp$ for zero: $x \perp 0,0 \perp x$ for all $x \in X$;

$\left(\mathrm{O}_{2}\right)$ independence: if $x, y \in X-\{0\}, x \perp y$, then $x, y$ are linearly independent;

$\left(O_{3}\right)$ homogeneity: if $x, y \in X, x \perp y$, then $\alpha x \perp \beta y$ for all $\alpha, \beta \in \mathbb{R}$;

(c) 2011 Park; licensee Springer. This is an Open Access article distributed under the terms of the Creative Commons Attribution License (http://creativecommons.org/licenses/by/2.0), which permits unrestricted use, distribution, and reproduction in any medium, provided the original work is properly cited. 
$\left(O_{4}\right)$ the Thalesian property: if $P$ is a 2-dimensional subspace of $X, x \in P$ and $\lambda \in \mathbb{R}_{+}$, which is the set of nonnegative real numbers, then there exists $y_{0} \in P$ such that $x \perp y_{0}$ and $x+y_{0} \perp \lambda x-y_{0}$.

The pair $(X, \perp)$ is called an orthogonality space. By an orthogonality normed space, we mean an orthogonality space having a normed structure.

Some interesting examples are

(i) The trivial orthogonality on a vector space $X$ defined by $\left(O_{1}\right)$, and for non-zero elements $x, y \in X, x \perp y$ if and only if $x, y$ are linearly independent.

(ii) The ordinary orthogonality on an inner product space $(X,\langle.,\rangle$.$) given by x \perp y$ if and only if $\langle x, y\rangle=0$.

(iii) The Birkhoff-James orthogonality on a normed space $(X,\|\cdot\|)$ defined by $x \perp y$ if and only if $\|x+\lambda y\| \geq\|x\|$ for all $\lambda \in \mathbb{R}$.

The relation $\perp$ is called symmetric if $x \perp y$ implies that $y \perp x$ for all $x, y \in X$. Clearly examples (i) and (ii) are symmetric but example (iii) is not. It is remarkable to note, however, that a real normed space of dimension greater than 2 is an inner product space if and only if the Birkhoff-James orthogonality is symmetric. There are several orthogonality notions on a real normed space such as Birkhoff-James, Boussouis, Singer, Carlsson, unitary-Boussouis, Roberts, Phythagorean, isosceles and Diminnie (see [6-12]).

The stability problem of functional equations originated from the following question of Ulam [13]: Under what condition does there exist an additive mapping near an approximately additive mapping? In 1941, Hyers [14] gave a partial affirmative answer to the question of Ulam in the context of Banach spaces. In 1978, Th.M. Rassias [15] extended the theorem of Hyers by considering the unbounded Cauchy difference $\| f(x+y)-f(x)$ $f(y) \| \leq \varepsilon\left(|| x||^{p}+|| y||^{p}\right),(\varepsilon>0, p \in[0,1))$. The result of Th.M. Rassias has provided a lot of influence in the development of what we now call generalized Hyers-Ulam stability or Hyers-Ulam stability of functional equations. During the last decades, several stability problems of functional equations have been investigated in the spirit of Hyers-Ulam-Rassias. The reader is referred to [16-20] and references therein for detailed information on stability of functional equations.

Ger and Sikorska [21] investigated the orthogonal stability of the Cauchy functional equation $f(x+y)=f(x)+f(y)$, namely, they showed that if $f$ is a mapping from an orthogonality space $X$ into a real Banach space $Y$ and $\mid f(x+y)-f(x)-f(y) \| \leq \varepsilon$ for all $x, y \in X$ with $x \perp y$ and some $\varepsilon>0$, then there exists exactly one orthogonally additive mapping $g: X \rightarrow Y$ such that $\|f(x)-g(x)\| \leq \frac{16}{3} \varepsilon$ for all $x \in X$.

The first author treating the stability of the quadratic equation was Skof [22] by proving that if $f$ is a mapping from a normed space $X$ into a Banach space $Y$ satisfying $\|f(x+y)+f(x-y)-2 f(x)-2 f(y)\| \leq \varepsilon$ for some $\varepsilon>0$, then there is a unique quadratic mapping $g: X \rightarrow Y$ such that $\|f(x)-g(x)\| \leq \frac{\varepsilon}{2}$. Cholewa [23] extended the Skof's theorem by replacing $X$ by an abelian group $G$. The Skof's result was later generalized by Czerwik [24] in the spirit of Hyers-Ulam-Rassias. The stability problem of functional equations has been extensively investigated by some mathematicians (see [25-28]).

The orthogonally quadratic equation

$$
f(x+y)+f(x-y)=2 f(x)+2 f(y), x \perp y
$$


was first investigated by Vajzović [29] when $X$ is a Hilbert space, $Y$ is the scalar field, $f$ is continuous and $\perp$ means the Hilbert space orthogonality. Later, Drljević [30], Fochi [31], Moslehian [32,33], Szabó [34], Moslehian and Th.M. Rassias [35] and Paganoni and Rätz [36] have investigated the orthogonal stability of functional equations.

Let $X$ be a set. A function $m: X \times X \rightarrow[0, \infty]$ is called a generalized metric on $X$ if $m$ satisfies

(1) $m(x, y)=0$ if and only if $x=y$;

(2) $m(x, y)=m(y, x)$ for all $x, y \in X$;

(3) $m(x, z) \leq m(x, y)+m(y, z)$ for all $x, y, z \in X$.

We recall a fundamental result in fixed point theory.

Theorem 1.1. $[37,38]$ Let $(X, m)$ be a complete generalized metric space and let $J: X \rightarrow$ $X$ be a strictly contractive mapping with Lipschitz constant $\alpha<1$. Then, for each given element $x \in X$, either

$$
m\left(J^{n} x, \quad J^{n+1} x\right)=\infty
$$

for all nonnegative integers $n$ or there exists a positive integer $n_{0}$ such that

(1) $m\left(J^{n} x, j^{n+1} x\right)<\infty, \forall n \geq n_{0}$;

(2) the sequence $\left\{J^{n} x\right\}$ converges to a fixed point $y^{*}$ of $J$;

(3) $y^{*}$ is the unique fixed point of $J$ in the set $Y=\left\{y \in X \mid m\left(J^{n_{0}} x, y\right)<\infty\right\}$;

(4) $m\left(y, y^{*}\right) \leq \frac{1}{1-\alpha} m(y, J y)$ for all $y \in Y$.

In 1996, Isac and Th.M. Rassias [39] were the first to provide applications of stability theory of functional equations for the proof of new fixed point theorems with applications. By using fixed point methods, the stability problems of several functional equations have been extensively investigated by a number of authors (see [40-46]).

This paper is organized as follows: In Section 2, we prove the Hyers-Ulam stability of the following orthogonally additive-quadratic functional equation

$$
2 f\left(\frac{x+y}{2}\right)+2 f\left(\frac{x-y}{2}\right)=\frac{3 f(x)}{2}-\frac{f(-x)}{2}+\frac{f(y)}{2}+\frac{f(-y)}{2}
$$

in orthogonality spaces by using the fixed point method. In Section 3, we prove the Hyers-Ulam stability of the orthogonally additive-quadratic functional equation (1.1) in orthogonality spaces by using the direct method.

Throughout this paper, assume that $(X, \perp)$ is an orthogonality space and that $\left(Y,\|.\| \|_{Y}\right)$ is a real Banach space.

\section{Hyers-Ulam Stability of the Orthogonally Additive-Quadratic Functional Equation (1.1): Fixed Point Method}

For a given mapping $f: X \rightarrow Y$, we define

$$
\begin{aligned}
D f(x, y): & =2 f\left(\frac{x+y}{2}\right)+2 f\left(\frac{x-y}{2}\right) \\
& -\frac{3 f(x)}{2}+\frac{f(-x)}{2}-\frac{f(y)}{2}-\frac{f(-y)}{2}
\end{aligned}
$$


for all $x, y \in X$ with $x \perp y$, where $\perp$ is the orthogonality in the sense of Rätz.

Let $f: X \rightarrow Y$ be an even mapping satisfying $f(0)=0$ and (1.1). Then, $f$ is a quadratic mapping, i.e., $2 f\left(\frac{x+y}{2}\right)+2 f\left(\frac{x-y}{2}\right)=f(x)+f(y)$ holds.

Using the fixed point method and applying some ideas from [18,21], we prove the Hyers-Ulam stability of the additive-quadratic functional equation $D f(x, y=0)$ in orthogonality spaces.

Theorem 2.1. Let $\phi: X^{2} \rightarrow[0, \infty)$ be a function such that there exists an $\alpha<1$ with

$$
\varphi(x, y) \leq 4 \alpha \varphi\left(\frac{x}{2}, \frac{y}{2}\right)
$$

for all $x, y \in X$ with $x \perp Y$. Let $f: X \rightarrow Y$ be an even mapping satisfying $f(0)=0$ and

$$
\|D f(x, y)\|_{Y} \leq \varphi(x, y)
$$

for all $x, y \in X$ with $x \perp y$. Then, there exists a unique orthogonally quadratic mapping $Q: X \rightarrow Y$ such that

$$
\|f(x)-Q(x)\|_{Y} \leq \frac{\alpha}{1-\alpha} \varphi(x, 0)
$$

for all $x \in X$.

Proof. Letting $y=0$ in (2.2), we get

$$
\left\|4 f\left(\frac{x}{2}\right)-f(x)\right\|_{Y} \leq \varphi(x, 0)
$$

for all $x \in X$, since $x \perp 0$. Thus

$$
\left\|f(x)-\frac{1}{4} f(2 x)\right\|_{Y} \leq \frac{1}{4} \varphi(2 x, 0) \leq \frac{4 \alpha}{4} \varphi(x, 0)
$$

for all $x \in X$.

Consider the set

$$
S:=\{h: X \rightarrow Y\}
$$

and introduce the generalized metric on $S$ :

$$
m(g, h)=\inf \left\{\mu \in \mathbb{R}_{+}:\|g(x)-h(x)\|_{Y} \leq \mu \varphi(x, 0), \forall x \in X\right\},
$$

where, as usual, $\inf \varphi=+\infty$. It is easy to show that $(S, m)$ is complete (see [[47], Lemma 2.1]).

Now we consider the linear mapping $J: S \rightarrow S$ such that

$$
J g(x):=\frac{1}{4} g(2 x)
$$

for all $x \in X$.

Let $g, h \in S$ be given such that $m(g, h)=\varepsilon$. Then,

$$
\|g(x)-h(x)\|_{Y} \leq \varphi(x, 0)
$$

for all $x \in X$. Hence

$$
\|J g(x)-J h(x)\|_{Y}=\left\|\frac{1}{4} g(2 x)-\frac{1}{4} h(2 x)\right\|_{Y} \leq \alpha \varphi(x, 0)
$$


for all $x \in X$. So $m(g, h)=\varepsilon$ implies that $m(J g, J h) \leq \alpha \varepsilon$. This means that

$$
m(J g, J h) \leq \alpha m(g, h)
$$

for all $g, h \in S$.

It follows from (2.5) that $m(f, J f) \leq \alpha$.

By Theorem 1.1, there exists a mapping $Q: X \rightarrow Y$ satisfying the following:

(1) $Q$ is a fixed point of $J$, i.e.,

$$
Q(2 x)=4 Q(x)
$$

for all $x \in X$. The mapping $Q$ is a unique fixed point of $J$ in the set

$$
M=\{g \in S: m(h, g)<\infty\} .
$$

This implies that $Q$ is a unique mapping satisfying (2.6) such that there exists a $\mu \in$ $(0, \infty)$ satisfying

$$
\|f(x)-Q(x)\|_{Y} \leq \mu \varphi(x, 0)
$$

for all $x \in X$;

(2) $m\left(J^{n} f, Q\right) \rightarrow 0$ as $n \rightarrow \infty$. This implies the equality

$$
\lim _{n \rightarrow \infty} \frac{1}{4^{n}} f\left(2^{n} x\right)=Q(x)
$$

for all $x \in X$;

(3) $m(f, Q) \leq \frac{1}{1-\alpha} m(f, J f)$, which implies the inequality

$$
m(f, Q) \leq \frac{\alpha}{1-\alpha}
$$

This implies that the inequality (2.3) holds.

It follows from (2.1) and (2.2) that

$$
\begin{aligned}
\|D Q(x, y)\|_{Y} & =\lim _{n \rightarrow \infty} \frac{1}{4^{n}}\left\|D f\left(2^{n} x, 2^{n} y\right)\right\|_{Y} \\
& \leq \lim _{n \rightarrow \infty} \frac{1}{4^{n}} \varphi\left(2^{n} x, 2^{n} y\right) \leq \lim _{n \rightarrow \infty} \frac{4^{n} \alpha^{n}}{4^{n}} \varphi(x, y)=0
\end{aligned}
$$

for all $x, y \in X$ with $x \perp y$. So $D Q(x, y)=0$ for all $x, y \in X$ with $x \perp y$. Hence $Q: X \rightarrow Y$ is an orthogonally quadratic mapping, as desired.

Corollary 2.2. Assume that $(X, \perp)$ is an orthogonality normed space. Let $\theta$ be a positive real number and $p$ a real number with $0<p<2$. Let $f: X \rightarrow Y$ be an even mapping satisfying $f(0)=0$ and

$$
\|D f(x, y)\|_{Y} \leq \theta\left(\|x\|^{p}+\|y\|^{p}\right)
$$

for all $x, y \in X$ with $x \perp y$. Then, there exists a unique orthogonally quadratic mapping $Q: X \rightarrow Y$ such that

$$
\|f(x)-Q(x)\|_{Y} \leq \frac{2^{p} \theta}{4-2^{p}}\|x\|^{p}
$$

for all $x \in X$.

Proof. Taking $\phi(x, y)=\theta\left(\|x\|^{p}+\|y\|^{p}\right)$ for all $x, y \in X$ with $x \perp y$ and choosing $\alpha=$ $2^{p-2}$ in Theorem 2.1, we get the desired result. 
Theorem 2.3. Let $\phi: X^{2} \rightarrow[0, \infty)$ be a function such that there exists an $\alpha<1$ with

$$
\varphi(x, y) \leq \frac{\alpha}{4} \varphi(2 x, 2 y)
$$

for all $x, y \in X$ with $x \perp y$. Let $f: X \rightarrow Y$ be an even mapping satisfying $f(0)=0$ and (2.2). Then, there exists a unique orthogonally quadratic mapping $Q: X \rightarrow Y$ such that

$$
\|f(x)-Q(x)\|_{Y} \leq \frac{1}{1-\alpha} \varphi(x, 0)
$$

for all $x \in X$.

Proof. Let $(S, m)$ be the generalized metric space defined in the proof of Theorem 2.1.

Now we consider the linear mapping $J: S \rightarrow S$ such that

$$
J g(x):=4 g\left(\frac{x}{2}\right)
$$

for all $x \in X$.

It follows from (2.4) that $m(f, J f) \leq 1$.

The rest of the proof is similar to the proof of Theorem 2.1.

Corollary 2.4. Assume that $(X, \perp)$ is an orthogonality normed space. Let $\theta$ be a positive real number and $p$ a real number with $p>2$. Let $f: X \rightarrow Y$ be an even mapping satisfying $f(0)=0$ and (2.7). Then, there exists a unique orthogonally quadratic mapping $Q: X \rightarrow Y$ such that

$$
\|f(x)-Q(x)\|_{Y} \leq \frac{2^{p} \theta}{2^{p}-4}\|x\|^{p}
$$

for all $x \in X$.

Proof. Taking $\phi(x, y)=\theta\left(\|x\|^{p}+\|y\|^{p}\right)$ for all $x, y \in X$ with $x \perp y$ and choosing $\alpha=$ $2^{2-p}$ in Theorem 2.3, we get the desired result.

Let $f: X \rightarrow Y$ be an odd mapping satisfying (1.1). Then, $f$ is an additive mapping, i.e., $2 f\left(\frac{x+y}{2}\right)+2 f\left(\frac{x-y}{2}\right)=2 f(x)$ holds.

Theorem 2.5. Let $\phi: X^{2} \rightarrow[0, \infty)$ be a function such that there exists an $\alpha<1$ with

$$
\varphi(x, y) \leq 2 \alpha \varphi\left(\frac{x}{2}, \frac{y}{2}\right)
$$

for all $x, y \in X$ with $x \perp y$. Let $f: X \rightarrow Y$ be an odd mapping satisfying (2.2). Then, there exists a unique orthogonally additive mapping $A: X \rightarrow Y$ such that

$$
\|f(x)-A(x)\|_{Y} \leq \frac{\alpha}{2-2 \alpha} \varphi(x, 0)
$$

for all $x \in X$.

Proof. Letting $y=0$ in (2.2), we get

$$
\left\|4 f\left(\frac{x}{2}\right)-2 f(x)\right\|_{Y} \leq \varphi(x, 0)
$$

for all $x \in X$, since $x \perp 0$. Thus,

$$
\left\|f(x)-\frac{1}{2} f(2 x)\right\|_{Y} \leq \frac{1}{4} \varphi(2 x, 0) \leq \frac{2 \alpha}{4} \varphi(x, 0)
$$


for all $x \in X$.

Let $(S, m)$ be the generalized metric space defined in the proof of Theorem 2.1.

Now we consider the linear mapping $J: S \rightarrow S$ such that

$$
J g(x):=\frac{1}{2} g(2 x)
$$

for all $x \in X$.

It follows from (2.9) that $m(f, J f) \leq \frac{\alpha}{2}$.

The rest of the proof is similar to the proof of Theorem 2.1.

Corollary 2.6. Assume that $(X, \perp)$ is an orthogonality normed space. Let $\theta$ be a positive real number and $p$ a real number with $0<p<1$. Let $f: X \rightarrow Y$ be an odd mapping satisfying (2.7). Then, there exists a unique orthogonally additive mapping $A: X \rightarrow Y$ such that

$$
\|f(x)-A(x)\|_{Y} \leq \frac{2^{p} \theta}{2\left(2-2^{p}\right)}\|x\|^{p}
$$

for all $x \in X$.

Proof. Taking $\phi(x, y)=\theta\left(\|x\|^{p}+\|y\|^{p}\right)$ for all $x, y \in X$ with $x \perp y$ and choosing $\alpha=$ $2^{p-1}$ in Theorem 2.5, we get the desired result.

Theorem 2.7. Let $\phi: X^{2} \rightarrow[0, \infty)$ be a function such that there exists an $\alpha<1$ with

$$
\varphi(x, y) \leq \frac{\alpha}{2} \varphi(2 x, 2 y)
$$

for all $x, y \in X$ with $x \perp y$. Let $f: X \rightarrow Y$ be an odd mapping satisfying (2.2). Then, there exists a unique orthogonally additive mapping $A: X \rightarrow Y$ such that

$$
\|f(x)-A(x)\|_{Y} \leq \frac{1}{2-2 \alpha} \varphi(x, 0)
$$

for all $x \in X$.

Proof. Let $(S, m)$ be the generalized metric space defined in the proof of Theorem 2.1. Now we consider the linear mapping $J: S \rightarrow S$ such that

$$
J g(x):=2 g\left(\frac{x}{2}\right)
$$

for all $x \in X$.

It follows from $(2.8)$ that $m(f, J f) \leq \frac{1}{2}$.

The rest of the proof is similar to the proof of Theorem 2.1.

Corollary 2.8. Assume that $(X, \perp)$ is an orthogonality normed space. Let $\theta$ be a positive real number and $p$ a real number with $p>1$. Let $f: X \rightarrow Y$ be an odd mapping satisfying (2.7). Then, there exists a unique orthogonally additive mapping $A: X \rightarrow Y$ such that

$$
\|f(x)-A(x)\|_{Y} \leq \frac{2^{p} \theta}{2\left(2^{p}-2\right)}\|x\|^{p}
$$

for all $x \in X$.

Proof. Taking $\phi(x, y)=\theta\left(\|x\|^{p}+\|y\|^{p}\right)$ for all $x, y \in X$ with $x \perp y$ and choosing $\alpha=$ $2^{1-p}$ in Theorem 2.7, we get the desired result. 
Let $f: X \rightarrow Y$ be a mapping satisfying $f(0)=0$ and (1.1). Let $f_{e}(x):=\frac{f(x)+f(-x)}{2}$ and $f_{o}(x)=\frac{f(x)-f(-x)}{2}$. Then, $f_{e}$ is an even mapping satisfying (1.1) and $f_{o}$ is an odd mapping satisfying (1.1) such that $f(x)=f_{e}(x)+f_{o}(x)$. So we obtain the following.

Theorem 2.9. Assume that $(X, \perp)$ is an orthogonality normed space. Let $\theta$ be a positive real number and $p$ a positive real number with $p \neq 1$. Let $f: X \rightarrow Y$ be a mapping satisfying $f(0)=0$ and (2.7). Then, there exist an orthogonally additive mapping $A: X \rightarrow Y$ and an orthogonally quadratic mapping $Q: X \rightarrow Y$ such that

$$
\|f(x)-A(x)-Q(x)\|_{Y} \leq\left(\frac{2^{p}}{2\left|2-2^{p}\right|}+\frac{2^{p}}{\left|4-2^{p}\right|}\right) \theta \|\left. x\right|^{p}
$$

for all $x \in X$.

\section{Hyers-Ulam Stability of the Orthogonally Additive-Quadratic Functional Equation (1.1): Direct Method}

In this section, using the direct method and applying some ideas from [18,21], we prove the Hyers-Ulam stability of the additive-quadratic functional equation $D f(x, y)=$ 0 in orthogonality spaces.

Theorem 3.1. Let $f: X \rightarrow Y$ be an even mapping satisfying $f(0)=0$ for which there exists a function $\phi: X^{2} \rightarrow[0, \infty)$ satisfying $(2.2)$ and

$$
\tilde{\varphi}(x, y):=\sum_{j=0}^{\infty} 4^{j} \varphi\left(\frac{x}{2^{j^{\prime}}}, \frac{y}{2^{j}}\right)<\infty
$$

for all $x, y \in X$ with $x \perp y$. Then, there exists a unique orthogonally quadratic mapping $Q: X \rightarrow Y$ such that

$$
\|f(x)-Q(x)\|_{Y} \leq \tilde{\varphi}(x, 0)
$$

for all $x \in X$.

Proof. It follows from (2.4) that

$$
\left\|4^{l} f\left(\frac{x}{2^{l}}\right)-4^{m} f\left(\frac{x}{2^{m}}\right)\right\|_{Y} \leq \sum_{j=1}^{m-1} 4^{j} \varphi\left(\frac{x}{2^{j}}, 0\right)
$$

for all nonnegative integers $m$ and $l$ with $m>l$ and all $x \in X$. It follows from (3.1) and (3.3) that the sequence $\left\{4^{n} f\left(\frac{x}{2^{n}}\right)\right\}$ is a Cauchy sequence for all $x \in X$. Since $Y$ is complete, the sequence $\left\{4^{n} f\left(\frac{x}{2^{n}}\right)\right\}$ converges. So one can define the mapping $Q: X \rightarrow Y$ by

$$
Q(x):=\lim _{n \rightarrow \infty} 4^{n} f\left(\frac{x}{2^{n}}\right)
$$

for all $x \in X$.

By the same reasoning as in the proof of Theorem 2.1, one can show that the mapping $Q: X \rightarrow Y$ is an orthogonally quadratic mapping satisfying (3.2).

Now, let $Q^{\prime}: X \rightarrow Y$ be another orthogonally quadratic mapping satisfying (3.2). Then, we have

$$
\begin{aligned}
\left\|Q(x)-Q^{\prime}(x)\right\|_{Y} & =4^{n}\left\|Q\left(\frac{x}{2^{n}}\right)-Q^{\prime}\left(\frac{x}{2^{n}}\right)\right\|_{Y} \\
\leq & 4^{n}\left(\left\|Q\left(\frac{x}{2^{n}}\right)-f\left(\frac{x}{2^{n}}\right)\right\|_{Y}+\left\|Q^{\prime}\left(\frac{x}{2^{n}}\right)-f\left(\frac{x}{2^{n}}\right)\right\|_{Y}\right) \\
\leq & 2 \cdot 4^{n} \tilde{\varphi}\left(\frac{x}{2^{n}}, 0\right),
\end{aligned}
$$


which tends to zero as $n \rightarrow \infty$ for all $x \in X$. So we can conclude that $Q(x)=Q^{\prime}(x)$ for all $x \in X$. This proves the uniqueness of $Q$.

Corollary 3.2. Assume that $(X, \perp)$ is an orthogonality space. Let $\theta$ be a positive real number and $p$ a real number with $p>2$. Let $f: X \rightarrow Y$ be an even mapping satisfying $f$ $(0)=0$ and (2.7). Then, there exists a unique orthogonally quadratic mapping $Q: X \rightarrow$ $Y$ such that

$$
\|f(x)-Q(x)\|_{Y} \leq \frac{2^{p} \theta}{2^{p}-4}\|x\|^{p}
$$

for all $x \in X$.

Proof. Taking $\phi(x, y)=\theta\left(|| x\left\|^{p}+\right\| y \|^{p}\right)$ for all $x, y \in X$ with $x \perp y$, and applying Theorem 3.1, we get the desired result.

Similarly, we can obtain the following. We will omit the proof.

Theorem 3.3. Let $f: X \rightarrow Y$ be an even mapping satisfying $f(0)=0$ for which there exists a function $\phi: X^{2} \rightarrow[0, \infty)$ satisfying $(2.2)$ and

$$
\tilde{\varphi}(x, y):=\sum_{j=1}^{\infty} \frac{1}{4^{j}} \varphi\left(2^{j} x, 2^{j} y\right)<\infty
$$

for all $x, y \in X$ with $x \perp y$. Then, there exists a unique orthogonally quadratic mapping $Q: X \rightarrow Y$ such that

$$
\|f(x)-Q(x)\|_{Y} \leq \tilde{\varphi}(x, 0)
$$

for all $x \in X$.

Corollary 3.4. Assume that $(X, \perp)$ is an orthogonality space. Let $\theta$ be a positive real number and $p$ a real number with $0<p<2$. Let $f: X \rightarrow Y$ be an even mapping satisfy$\operatorname{ing} f(0)=0$ and (2.7). Then, there exists a unique orthogonally quadratic mapping $Q$ : $X \rightarrow Y$ such that

$$
\|f(x)-Q(x)\|_{Y} \leq \frac{2^{p} \theta}{4-2^{p}}\|x\|^{p}
$$

for all $x \in X$.

Proof. Taking $\phi(x, y)=\theta\left(\|x\|^{p}+\|y\|^{p}\right)$ for all $x, y \in X$ with $x \perp y$, and applying Theorem 3.3, we get the desired result.

Theorem 3.5. Let $f: X \rightarrow Y$ be an odd mapping for which there exists a function $\phi$ : $X^{2} \rightarrow[0, \infty)$ satisfying $(2.2)$ and

$$
\tilde{\varphi}(x, y):=\sum_{j=0}^{\infty} 2^{j} \varphi\left(\frac{x}{2^{j^{\prime}}}, \frac{y}{2^{j}}\right)<\infty
$$

for all $x, y \in X$ with $x \perp y$. Then, there exists a unique orthogonally additive mapping $A: X \rightarrow Y$ such that

$$
\|f(x)-A(x)\|_{Y} \leq \frac{1}{2} \tilde{\varphi}(x, 0)
$$

for all $x \in X$.

Proof. It follows from (2.8) that

$$
\left\|f(x)-2 f\left(\frac{x}{2}\right)\right\|_{Y} \leq \frac{1}{2} \varphi(x, 0)
$$


for all $x \in X$.

The rest of the proof is similar to the proofs of Theorems 2.5 and 3.1.

Corollary 3.6. Assume that $(X, \perp)$ is an orthogonality space. Let $\theta$ be a positive real number and $p$ a real number with $p>1$. Let $f: X \rightarrow Y$ be an odd mapping satisfying (2.7). Then, there exists a unique orthogonally additive mapping $A: X \rightarrow Y$ such that

$$
\|f(x)-A(x)\|_{Y} \leq \frac{2^{p} \theta}{2\left(2^{p}-2\right)}\|x\|^{p}
$$

for all $x \in X$.

Proof. Taking $\phi(x, y)=\theta\left(\|x\|^{p}+\|y\|^{p}\right)$ for all $x, y \in X$ with $x \perp y$, and applying Theorem 3.5, we get the desired result.

Similarly, we can obtain the following. We will omit the proof.

Theorem 3.7. Let $f: X \rightarrow Y$ be an odd mapping for which there exists a function $\phi$ : $X^{2} \rightarrow[0, \infty)$ satisfying $(2.2)$ and

$$
\tilde{\varphi}(x, y):=\sum_{j=1}^{\infty} \frac{1}{2^{j}} \varphi\left(2^{j} x, 2^{j} y\right)<\infty
$$

for all $x, y \in X$ with $x \perp y$. Then, there exists a unique orthogonally additive mapping $A: X \rightarrow Y$ such that

$$
\|f(x)-A(x)\|_{Y} \leq \frac{1}{2} \tilde{\varphi}(x, 0)
$$

for all $x \in X$.

Corollary 3.8. Assume that $(X, \perp)$ is an orthogonality space. Let $\theta$ be a positive real number and $p$ a real number with $0<p<1$. Let $f: X \rightarrow Y$ be an odd mapping satisfying (2.7). Then, there exists a unique orthogonally additive mapping $A: X \rightarrow Y$ such that

$$
\|f(x)-A(x)\|_{Y} \leq \frac{2^{p} \theta}{2\left(2-2^{p}\right)}\|x\|^{p}
$$

for all $x \in X$.

Proof. Taking $\phi(x, y)=\theta\left(\|x\|^{p}+\|y\|^{p}\right)$ for all $x, y \in X$ with $x \perp y$, and applying Theorem 3.7 , we get the desired result.

\section{Competing interests}

The author declares that they have no competing interests.

Received: 17 March 2011 Accepted: 25 October 2011 Published: 25 October 2011

References

1. Pinsker, AG: Sur une fonctionnelle dans I'espace de Hilbert. In: DokI CR (ed.) Acad Sci URSS n Ser. 20, 411-414 (1938)

2. Sundaresan, K: Orthogonality and nonlinear functionals on Banach spaces. Proc Amer Math Soc. 34, 187-190 (1972). doi:10.1090/S0002-9939-1972-0291835-X

3. Gudder, S, Strawther, D: Orthogonally additive and orthogonally increasing functions on vector spaces. Pac J Math. 58, 427-436 (1975)

4. Rätz, J: On orthogonally additive mappings. Aequationes Math. 28, 35-49 (1985). doi:10.1007/BF02189390

5. Rätz, J, Szabó, Gy: On orthogonally additive mappings IV. Aequationes Math. 38, 73-85 (1989). doi:10.1007/BF01839496

6. Alonso, J, Benítez, C: Orthogonality in normed linear spaces: a survey I. Main properties. Extr Math. 3, 1-15 (1988)

7. Alonso, J, Benítez, C: Orthogonality in normed linear spaces: a survey II. Relations between main orthogonalities. Extr Math. 4, 121-131 (1989)

8. Birkhoff, G: Orthogonality in linear metric spaces. Duke Math J. 1, 169-172 (1935). doi:10.1215/S0012-7094-35-00115-6

9. Carlsson, SO: Orthogonality in normed linear spaces. Ark Mat. 4, 297-318 (1962). doi:10.1007/BF02591506 
10. Diminnie, CR: A new orthogonality relation for normed linear spaces. Math Nachr. 114, 197-203 (1983). doi:10.1002/ mana. 19831140115

11. James, RC: Orthogonality in normed linear spaces. Duke Math J. 12, 291-302 (1945). doi:10.1215/S0012-7094-45-01223-3

12. James, RC: Orthogonality and linear functionals in normed linear spaces. Trans Amer Math Soc. 61, 265-292 (1947). doi:10.1090/50002-9947-1947-0021241-4

13. Ulam, SM: Problems in Modern Mathematics. Wiley, New York (1960)

14. Hyers, DH: On the stability of the linear functional equation. Proc Natl Acad Sci USA. 27, $222-224$ (1941). doi:10.1073/ pnas.27.4.222

15. Rassias, ThM: On the stability of the linear mapping in Banach spaces. Proc Amer Math Soc. 72, 297-300 (1978). doi:10.1090/S0002-9939-1978-0507327-1

16. Czerwik, S: Functional Equations and Inequalities in Several Variables. World Scientific Publishing Company, New Jersey (2002)

17. Czerwik, S: Stability of Functional Equations of Ulam-Hyers-Rassias Type. Hadronic Press, Palm Harbor (2003)

18. Hyers, DH, Isac, G, Rassias, ThM: Stability of Functional Equations in Several Variables. Birkhäuser, Basel (1998)

19. Jung, S: Hyers-Ulam-Rassias Stability of Functional Equations in Mathematical Analysis. Hadronic Press, Palm Harbor (2001)

20. Rassias, ThM, ed: Functional Equations, Inequalities and Applications. Kluwer, Dordrecht (2003)

21. Ger, R, Sikorska, J: Stability of the orthogonal additivity. Bull Polish Acad Sci Math. 43, 143-151 (1995)

22. Skof, F: Proprietà locali e approssimazione di operatori. Rend Sem Mat Fis Milano. 53, 113-129 (1983). doi:10.1007/ BF02924890

23. Cholewa, PW: Remarks on the stability of functional equations. Aequationes Math. 27, 76-86 (1984). doi:10.1007/ BF02192660

24. Czerwik, S: On the stability of the quadratic mapping in normed spaces. Abh Math Sem Univ Hamburg. 62, 59-64 (1992). doi:10.1007/BF02941618

25. Park, C, Park, J: Generalized Hyers-Ulam stability of an Euler-Lagrange type additive mapping. J Differ Equat Appl. 12, 1277-1288 (2006). doi:10.1080/10236190600986925

26. Rassias, ThM: On the stability of the quadratic functional equation and its applications. pp. 89-124. Studia Univ BabeşBolyai Math43, (1998)

27. Rassias, ThM: The problem of S.M. Ulam for approximately multiplicative mappings. J Math Anal Appl. 246, 352-378 (2000). doi:10.1006/jmaa.2000.6788

28. Rassias, ThM: On the stability of functional equations in Banach spaces. J Math Anal Appl. 251, 264-284 (2000). doi:10.1006/jmaa.2000.7046

29. Vajzović, F: Über das Funktional $H$ mit der Eigenschaft: $(x, y)=0 \otimes H(x+y)+H(x-y)=2 H(x)+2 H(y)$. Glasnik Mat Ser III. 2(22), 73-81 (1967)

30. Drljević, F: On a functional which is quadratic on A-orthogonal vectors. Publ Inst Math (Beograd). 54, 63-71 (1986)

31. Fochi, M: Functional equations in A-orthogonal vectors. Aequationes Math. 38, 28-40 (1989). doi:10.1007/BF01839491

32. Moslehian, MS: On the orthogonal stability of the Pexiderized quadratic equation. J Differ Equat Appl. 11, 999-1004 (2005). doi:10.1080/10236190500273226

33. Moslehian, MS: On the stability of the orthogonal Pexiderized Cauchy equation. J Math Anal Appl. 318, 211-223 (2006). doi:10.1016/j.jmaa.2005.05.052

34. Szabó, Gy: Sesquilinear-orthogonally quadratic mappings. Aequationes Math. 40, 190-200 (1990). doi:10.1007/ BF02112295

35. Moslehian, MS, Rassias, ThM: Orthogonal stability of additive type equations. Aequationes Math. 73, 249-259 (2007). doi:10.1007/s00010-006-2868-0

36. Paganoni, L, Rätz, J: Conditional function equations and orthogonal additivity. Aequationes Math. 50, 135-142 (1995). doi:10.1007/BF01831116

37. Cădariu, L, Radu, V: Fixed points and the stability of Jensen's functional equation. J Inequal Pure Appl Math. 4(1), Art. ID 4 (2003)

38. Diaz, J, Margolis, B: A fixed point theorem of the alternative for contractions on a generalized complete metric space. Bull Amer Math Soc. 74, 305-309 (1968). doi:10.1090/S0002-9904-1968-11933-0

39. Isac, G, Rassias, ThM: Stability of $\psi$-additive mappings: applications to nonlinear analysis. Intern J Math Math Sci. 19 , 219-228 (1996). doi:10.1155/S0161171296000324

40. Cădariu, L, Radu, V: On the stability of the Cauchy functional equation: a fixed point approach. Grazer Math Ber. 346, 43-52 (2004)

41. Cădariu, L, Radu, V: Fixed point methods for the generalized stability of functional equations in a single variable. Fixed Point Theory Appl. 2008, Art ID 749392 (2008)

42. Jung, Y, Chang, l: The stability of a cubic type functional equation with the fixed point alternative. J Math Anal Appl. 306, 752-760 (2005). doi:10.1016/j.jmaa.2004.10.017

43. Mirzavaziri, M, Moslehian, MS: A fixed point approach to stability of a quadratic equation. Bull Braz Math Soc. 37, 361-376 (2006). doi:10.1007/s00574-006-0016-z

44. Park, C: Fixed points and Hyers-Ulam-Rassias stability of Cauchy-Jensen functional equations in Banach algebras. Fixed Point Theory Appl. 2007, Art ID 50175 (2007)

45. Park, C: Generalized Hyers-Ulam-Rassias stability of quadratic functional equations: a fixed point approach. Fixed Point Theory Appl. 2008, Art ID 493751 (2008)

46. Radu, V: The fixed point alternative and the stability of functional equations. Fixed Point Theory. 4, 91-96 (2003)

47. Miheț, $\mathrm{D}, \mathrm{Radu}, \mathrm{V}$ : On the stability of the additive Cauchy functional equation in random normed spaces. J Math Anal Appl. 343, 567-572 (2008)

doi:10.1186/1687-1812-2011-66

Cite this article as: Park: Orthogonal Stability of an Additive-Quadratic Functional Equation. Fixed Point Theory and Applications 2011 2011:66. 